\title{
Analysis of capnogram during patient-controlled sedation with propofol or midazolam in healthy volunteers
}

\author{
Jung-Man Lee1, ${ }^{1}$ wang-Suk Seo ${ }^{2}$, Hyun Jeong Kim² \\ 1 Department of Anesthesiology and Pain Medicine, Seoul Metropolitan Government-Seoul National University Boramae \\ Medical Center, Seoul, Korea, Republic of \\ 2 Department of Dental Anesthesiology, Seoul National University Dental Hospital, Seoul, Korea, Republic of
}

\section{Background}

Despite being an important technique to prevent respiratory problems during sedation, capnography has some limitation in detecting respiratory depression. Thus, a more accurate capnography monitoring algorithm is needed. We analyzed capnograms obtained from the nasal cannulas of healthy volunteers during patient-controlled sedation (PCS) with propofol or midazolam.

\section{Methods}

$\diamond$ The study was approved by the IRB.

$\diamond$ Sixty healthy participants were randomly assigned to one of six groups, according to the drug and dose used in PCS.

$\diamond$ Digital data on the partial pressure of carbon dioxide before and during PCS were converted into analyzable waves.

$\diamond$ We calculated the capnographic inspiratory and expiratory times using the peak velocity of increasing and decreasing $\mathrm{PCO}_{2}$

$\diamond$ The ratio "[capnographic inspirtory time]/[capnographic expiratory time] was defined as "capnographic I:E ratio in this study.

$\diamond$ We determined the capnogrphic I:E ratio before PCS bagan (before PCS) and during PCS while the paricipant was conscious or unconscious.

$\diamond$ Respiratory depression event was defined by the absence of waves in the capnogram for $>30 \mathrm{~s}$ or an event of peripheral oxygen saturation $<90 \%$.

$\diamond$ We used LoFloTM $\mathrm{CO}_{2}$ sampling $\mathrm{O}_{2}$ delivery nasal cannula (Respironics Co. Inc, MN, US).

$\diamond$ We used the MATLAB ${ }^{\circledR}$ program for analysis of capnogram.

Figure 1. Process of calculationg capnographic I:E ratio using MATLAB program
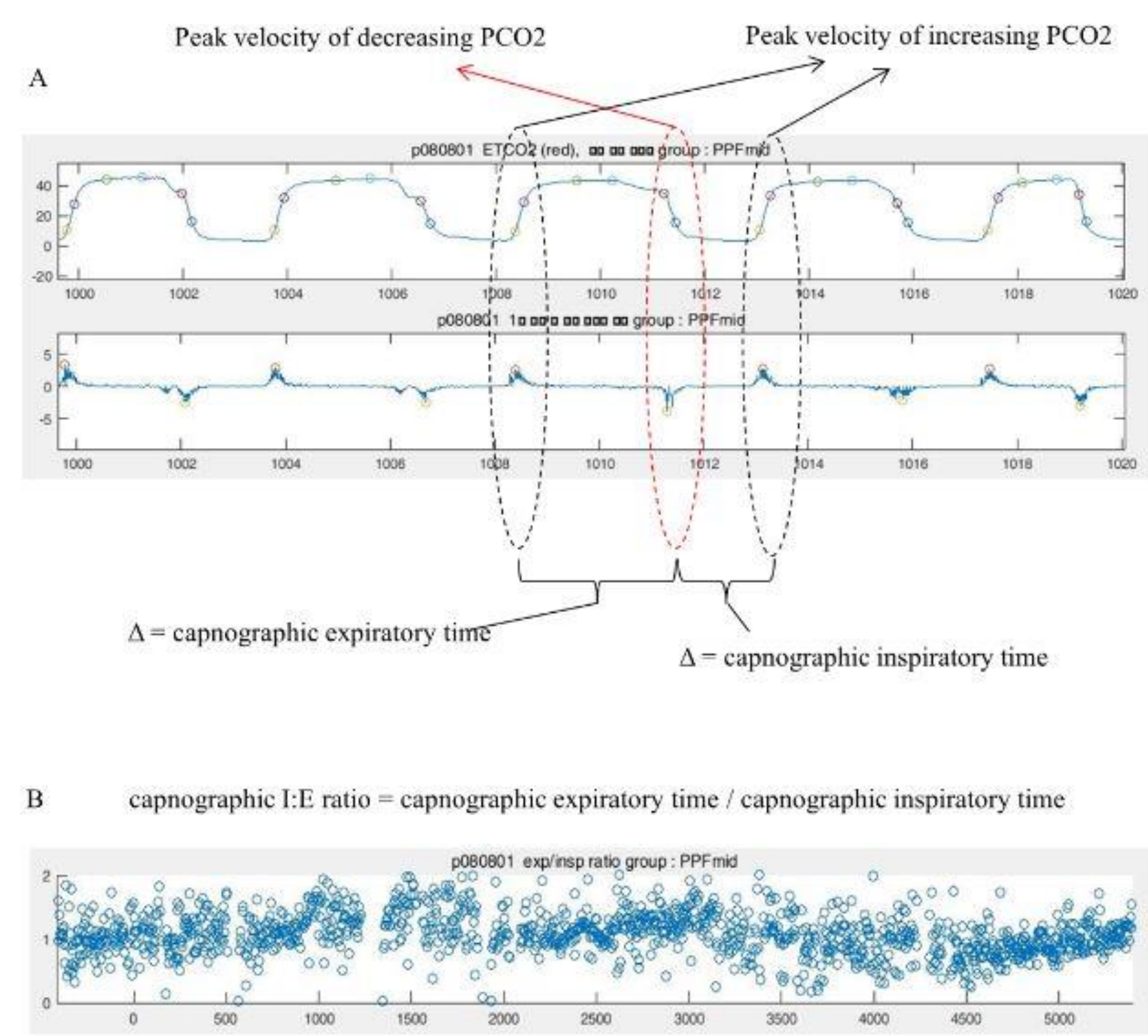

Results

$\diamond$ Capnographic I:E ratio was lower during the 'unconsciousness during PCS' (mean: 0.93, 95\% CI: 0.89-0.98) than it was in the 'before PCS' $(1.10,1.04-1.15)$ or 'consciousness during PCS' $(1.09,1.03-1.15)$ $(\mathrm{p}<0.001)$. [Figure 2]

In 21 participants who experienced respiratory depression (RD), capnographic I:E ratios tends to disperse immediately before a RD event compared to the normal breathing period, as evidenced by the significantly higher average standard deviations during these events (during RD: $0.71 \pm 0.69$, normal breathing: $0.17 \pm 0.10, p=0.002$ ) [Table]

Figure 2. Capnographic I:E ratio according to patient-controlled sedation and mental status

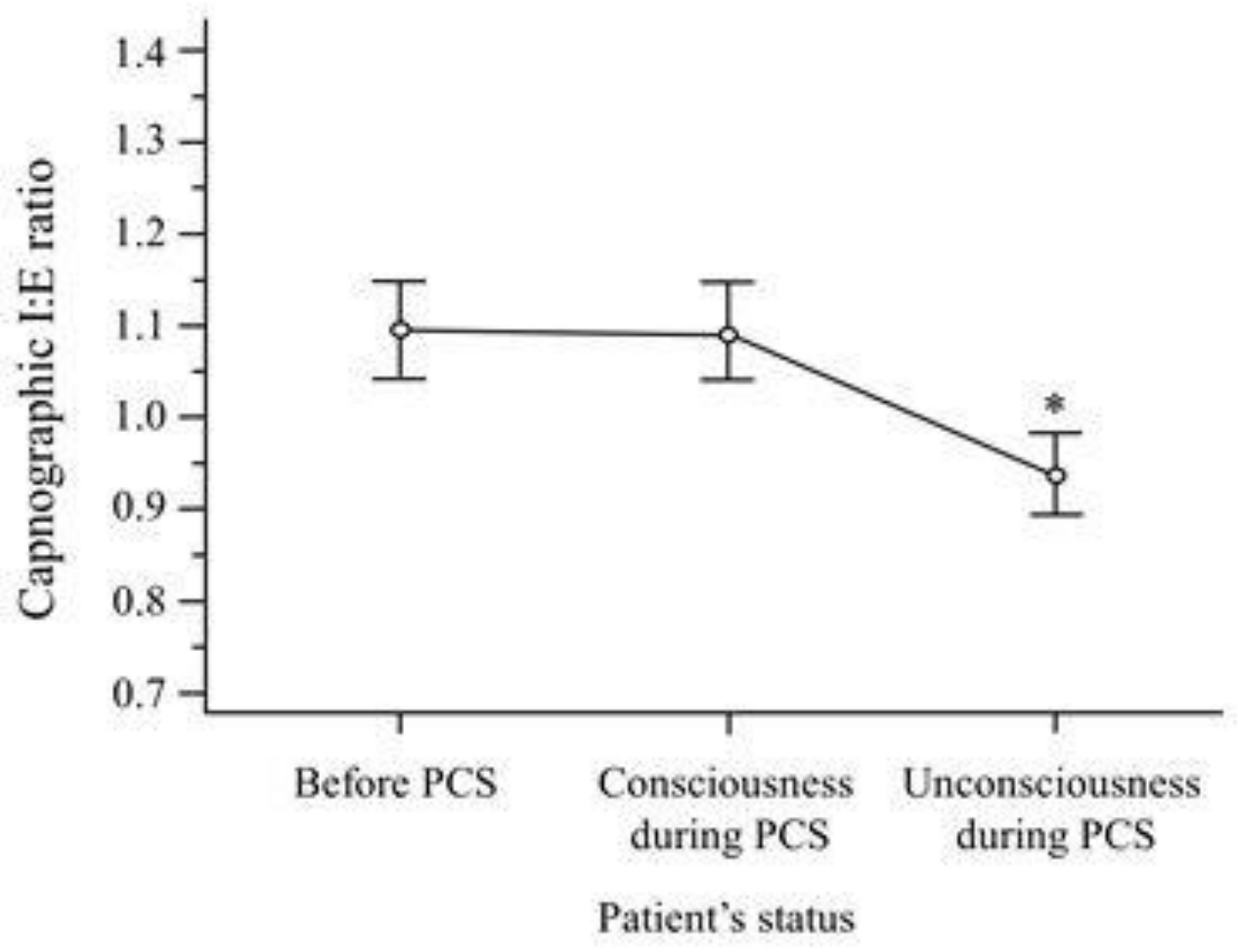

Table. Comparison of mean and standard deviation of capnographic I:E ratio between normal breathing status and respiratory depression (RD) status in 21 patients experienced $\mathrm{RD}$

\begin{tabular}{cccc}
\hline \multirow{2}{*}{$\begin{array}{c}\text { during normal } \\
\text { breathing }\end{array}$} & during RD & $P$ \\
\hline Mean & $0.96 \pm 0.24$ & $1.02 \pm 0.28$ & 0.515 \\
Standard deviation & $0.17 \pm 0.10$ & $0.71 \pm 0.69$ & 0.002 \\
\hline
\end{tabular}

Conclusion

Quantification analysis of capnograms could be used to improve diagnosis and prediction of respiratory depression and desaturation events and, therefore, could be a useful tool for monitoring patients during sedation. 